\title{
Silicon Nanophotonic Waveguide Circuits and Devices
}

\author{
Wim Bogaerts, Pieter Dumon, Shankar Kumar Selvaraja, Dries Van Thourhout and Roel Baets \\ IMEC - Ghent University, Department of Information Technology, 9000 Gent, Belgium \\ Email: wim.bogaerts@intec.ugent.be
}

\begin{abstract}
Silicon on Insulator is an ideal platform for largescale nanophotonic integration. We show that tight process control is needed for well-functioning filters, and discuss a number of devices based on these filters.
\end{abstract}

\section{INTRODUCTION}

Silicon on Insulator (SOI) has already proven itself as a very suitable material for integrated photonics [1]. Not only because of the high refractive index contrast, which allows for submicron waveguides and sharp bends with radii of $3 \mu \mathrm{m}$ or less [2], but also because the material can be processed with the same tool set as used for electronics manufacturing. This combination, a small footprint and an a potential industrial manufacturing base, gives silicon photonics the scalability needed for low-cost, large-volume applications.

We use these photonic wires to implement a variety of wavelength-selective components. These include ring resonators, but also arrayed waveguide gratings scaled to very small footprints. However, the submicron dimensions of the photonic wires make these filters exceptionally sensitive to process variations. While in the end there will always be the need for a tuning mechanism, good process control can already bring the device very close to its desired specification. This, in turn, reduces the power consumption required for tuning. We show that this is possible with classical CMOS technology. Finally, we also discuss a number of device concepts which can only become viable through SOI nanophotonics, because of cost and volume considerations.

\section{Passive WaVelength-Selective COMPONENTS}

The high contrast photonic wires in SOI allow for very sharp bends, which in turn reduces the footprint of many common waveguide components. Because of that, wavelength selective elements such as ring resonators can be made very compact, thus they can have a very large free spectral range [2]. Also, the relatively strong dispersion of photonic wires $\left(n_{g}\right.$ 4.0) allows for shorter delay lines. This, in combination with acceptable propagation losses of a few $\mathrm{dB} / \mathrm{cm}$ [3], [1], makes photonic wires ideal to construct wavelength selective components.

A result of this is shown in Fig. 1. This $8 \times 400 \mathrm{GHz}$ arrayed waveguide grating (AWG) has a footprint of only $200 \times 350 \mu \mathrm{m}^{2}$. Because of the tight process control (discussed in the next section) the path length difference in the arms is well controlled, resulting in a low crosstalk level of $-25 d B$; the lowest reported in a demultiplexer device of this size with

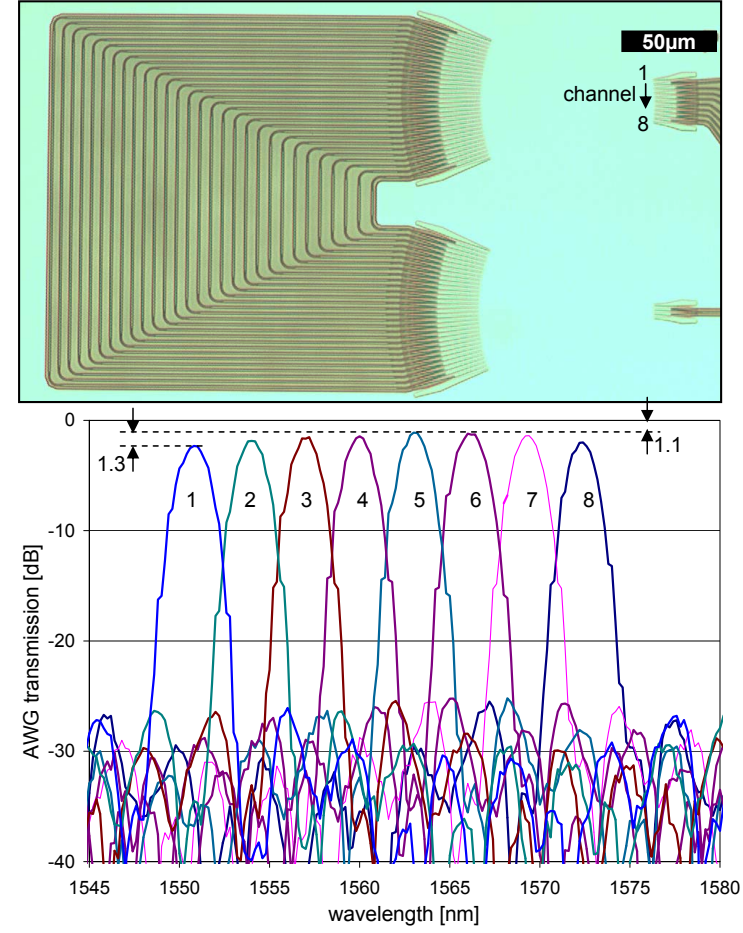

Fig. 1. Compact 8-channel arrayed waveguide grating

a similar channel spacing. Also, by using a combination of deep and shallow etch layers [2], the insertion loss of the entire AWG is only $1.1 \mathrm{~dB}$.

\section{Process Control}

With waveguide dimensions of $500 \mathrm{~nm}$, photonic wires fall well within the fabrication capabilities of high-end CMOS manufacturing tools. However, the fabrication tolerances of photonic components are much more strict that for electronics, where margins of $5 \%$ or $10 \%$ are acceptable. For wavelengthselective filters, a deviation of waveguide width of $1 \mathrm{~nm}$ will correspond to approximately $1 \mathrm{~nm}$ wavelength shift. While such a shift can be compensated for by thermal or other tuning mechanisms, the acceptable tolerances are still of the order of $1 \%$. However, we have demonstrated that with reasonable process control, these tolerances can be met with standard CMOS fabrication tools [4]. 


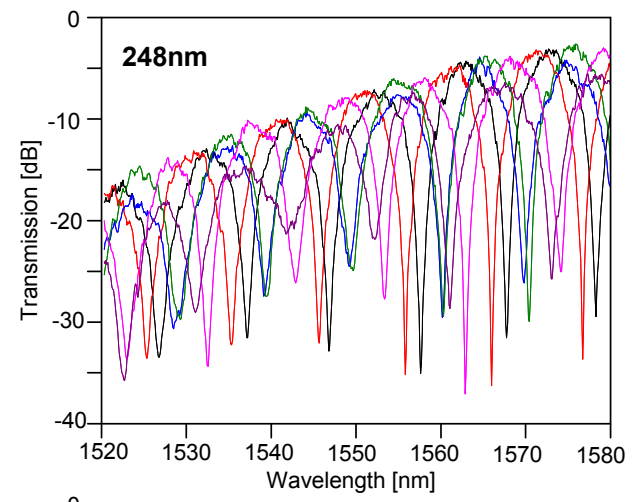

0

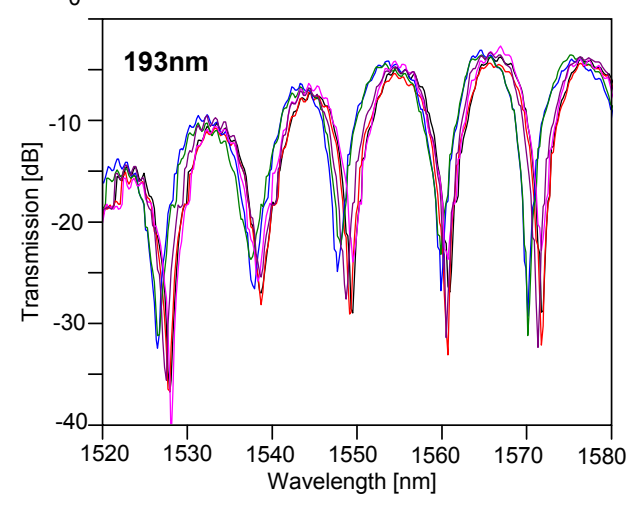

Fig. 2. Transmission of 6 nominally identical Mach-Zehnder interferometers. Top: made with $248 \mathrm{~nm}$ deep UV lithography. Bottom: with $193 \mathrm{~nm}$.

One of the critical steps in the fabrication process is the lithography. While for research purposs e-beam lithography is attractive for its flexibility and high resolution, we make use of standard CMOS tools, based on optical projection lithography at deep UV wavelengths $(248 \mathrm{~nm}$ and $193 \mathrm{~nm})$. The resolution of these tools is typically less than that of high-end e-beam lithography, and this is not only reflected in final device geometry, but also in the uniformity and reproducibility of the fabrication process. This is illustrated in Fig. 2, where we plotted the transmission of 6 nominally identical Mach-Zehnder interferometers on a single chip. The design was fabricated from the same mask plate both with $248 \mathrm{~nm}$ and $193 \mathrm{~nm}$ lithography. It is clear that the highest resolution process gives the best uniformity, with deviations of the transmission dip wavelength of approximately $1 \mathrm{~nm}$.

\section{PHOTONIC WIRE DEVICES}

Wavelength-selective components can be used for various applications. In communications, wavelength division multiplexing is the obvious candidate, and in its simplest form, a very low-cost duplexing system based on SOI photonic waveguides could drive wide deployment of fiber access networks [5]. The WDM principle could also be applied for wavelength routing of high-speed signals between chips or boards in highperformance computing systems [6]. When used together with fiber arrays, the actual chip of the routing backplane, has a smaller footprint than the connector, allowing for an elegant packaging approach [7].

The sharp wavelength response of a ring resonator can also be applied for optical sensors. The group index of the waveguide can be changed modified by an outside influence, such as mechanical stress, temperature or the presence of specific molecules in the cladding material. Especially the latter property is interesting. In SOI photonic wires, about $20 \%$ of the optical power is located in the top cladding of the waveguide (either air or an overlay material). With a ring resonator one can measure index changes smaller than $10^{-4}$. In addition, by chemically activating the surface of the ring the sensor can be made extremely sensitive to specific biomolecules, which enables bacterial and virus detection. In such miniaturized label-free biosensors the light can feel the change in the top cladding when biomolecules attach to a chemically functionalized layer above the resonator [8].

\section{CONCLUSION}

SOI Photonic wires allow for very compact wavelength selective functions, which can be used in a variety of devices, from telecommunications to biosensing. Modern CMOS fabrication technologies now allow sufficient process control to meet the strict tolerances required for such applications.

\section{ACKNOWLEDGMENT}

Part of this work was supported by the European Union through the IST-PICCO, IST-PICMOS and ICT WaDiMOS project and the IST-ePIXnet Network of Excellence. Part of this work was supported by the Belgian Science Policy IAPPHOTONnetwork and IAP-photonics@be projects, and the Institute for the Promotion of Innovation through Science and Technology in Flanders (IWT-Vlaanderen) under the SBO epSOC project.

\section{REFERENCES}

[1] W. Bogaerts, R. Baets, P. Dumon, V. Wiaux, S. Beckx, D. Taillaert, B. Luyssaert, J. Van Campenhout, P. Bienstman, and D. Van Thourhout, "Nanophotonic waveguides in Silicon-on-insulator fabricated with CMOS technology," J. Lightwave Technol., vol. 23, no. 1, pp. 401-412, 2005.

[2] W. Bogaerts, P. Dumon, D. Van Thourhout, D. Taillaert, P. Jaenen, J. Wouters, B. S., V. Wiaux, and R. Baets, "Compact wavelength-selective functions in silicon-on-insulator photonic wires," J. Sel. Top. Quantum Electron., vol. 12, no. 6, pp. 1394-1401, December 2006.

[3] Y. A. Vlasov and S. McNab, "Losses in single-mode silicon-on-insulator strip waveguides and bends," Opt. Express, vol. 12, no. 8, pp. 1622 1631, April 2004.

[4] S. Selvaraja, W. Bogaerts, D. Van Thourhout, and R. Baets, "Fabrication of uniform photonic devices using $193 \mathrm{~nm}$ optical lithography in siliconon-insulator,' Proc. ECIO, p. FrB3, 2008.

[5] G. Roelkens, D. Van Thourhout, and R. Baets, "Silicon-on-insulator ultacompact duplexer based on a diffractive grating structure," Opt. Express, vol. 15, no. 16, p. 10091, 2007.

[6] P. Dumon, W. Bogaerts, D. Van Thourhout, D. Taillaert, R. Baets, J. Wouters, S. Beckx, and P. Jaenen, "Compact wavelength router based on a silicon-on-insulator arrayed waveguide grating pigtailed to a fiber array," Opt. Express, vol. 14, no. 2, pp. 664-669, January 2006.

[7] L. Zimmermann, H. Schroeder, W. Bogaerts, P. Dumon, and T. Tekin, "epixpack - advanced smart packaging solutions for silicon photonics," Proc. ECIO, p. WeB2, 2008.

[8] K. De Vos, I. Bartolozzi, E. Schacht, P. Bienstman, and R. Baets, "Siliconon-insulator microring resonator for sensitive and label-free biosensing," Opt. Express, vol. 15, no. 12, pp. 7610-7615, 2007. 